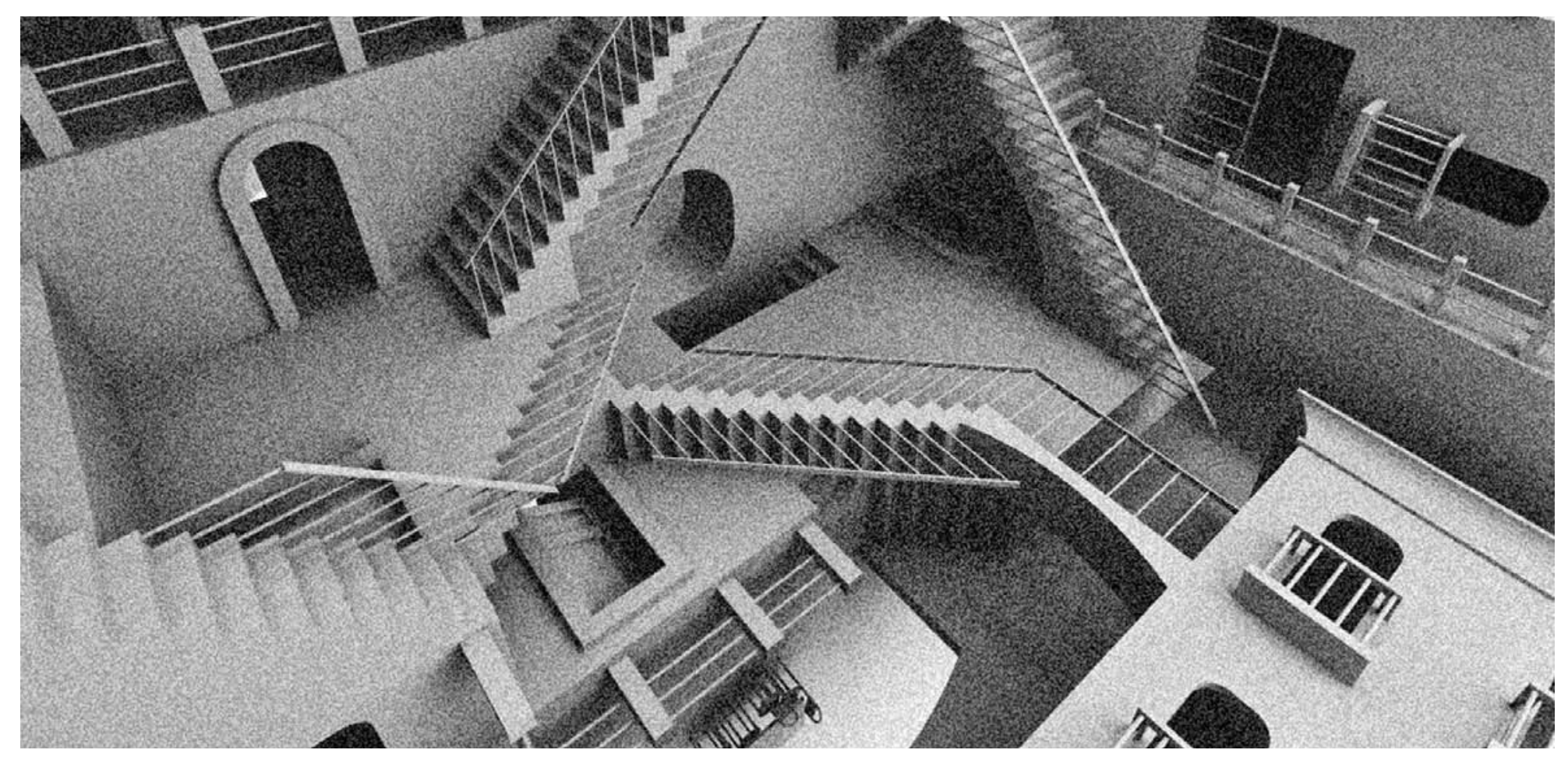

\title{
Diseño web y arquitectura de información para sitios 2.0
}

\section{Web Design and Information Architecture for 2.0 Sites}

Claudia Gutiérrez, KeplerMedia S.A. Santiago, Chile (cgutierhlayahoo.com)

Recibido: 4/5/2008/Aceptado:21/7/2008

I Si en una primera fase de la web los sitios eran meras réplicas ฮ de brochures corporativos, la tendencia actual a la producción

E y diseminación de información a través de herramientas de web

o 2.0 tales como blogs o redes sociales, apunta a que el contenido

$\approx$ se ha transformado en un protagonista privilegiado. Desde esa perspectiva, ha surgido la necesidad de que los proyectos digitales dispongan de un orden lógico que emane de pautas que no sólo permitan jerarquizar los textos, sino también los elementos gráficos expuestos. De este modo, un diseño y arquitectura de información adecuados deben no sólo construirse en base a una estructuración racional, sino también tomar en cuenta factores "emocionales" relativos a la experiencia de su uso. Solo la combinación de ambos permite que los usuarios se sientan cómodos, antes, durante y después de haber visitado un sitio web.

Palabras clave: diseño web, arquitectura de información, interfaces digitales, internet.
In the first stage of the web its sites were replicas of corporate brochures, $\underset{\sigma}{ \pm}$ while the current trend of production and dissemination of information through the tools of the web 2.0, such as blogs or social networking sites, sugin gest that the content has become the privileged player. From that perspective, $\varangle$ there is a need of having an order based on guidelines that determine both the hierarchy of texts and the layout of graphic elements. Therefore, efficient design and architecture of information should be built not only from an exclusively rational structure, but also take into account emotional elements relative to the experience of its use, mainly through its design. Only the combination of both enable users to feel comfortable before, during and after having visited a website.

Keywords: web design, information architecture, digital interfaces, Internet. 
$\mathbf{P}$ rece bastante improbable que el Catálogo de objetos imposibles que el artista francés Jacques Carelman¹ lanzó en 1969 como parodia a los productos de venta por catálogo, tenga absoluta vigencia hoy. Su trabajo, además del valor artístico que tiene, es reconocido porque sus piezas han permitido ejemplificar uno de los principios de la comunicación en el campo de los multimedios interactivos: la usabilidad.

El investigador Donald Norman usa la imagen de la célebre cafetera para masoquistas de Carelman para ilustrar la portada de su libro El diseño de objetos cotidianos donde revisa la relación entre el uso y la interfaz de muchas de las cosas que usamos día a día. La cafetera constaba de todos los elementos que debería tener una tal como la conocemos desde su diseño inicial: tapa, asa y boca. Sin embargo, todos estos elementos se encontraban alineados en un mismo sentido. De ahí su nombre. La cafetera era para masoquistas, pues se volvía físicamente imposible utilizarla.

\section{La cafetera para masoquistas de Carelman}

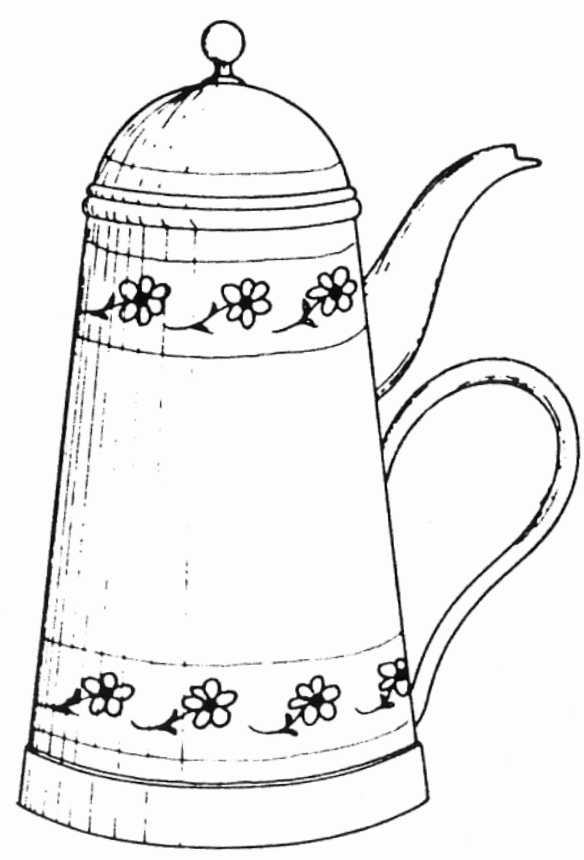

fuente: Norman, D.A. $(1990)$
Es precisamente en la usabilidad donde se establece el vínculo entre la cafetera de Carelman y el diseño de interfaces digitales. En relación a esto y a su aplicación en páginas web, Jakob Nielsen (2003) define a la usabilidad como "el atributo de calidad que mide la facilidad en el uso de las interfaces. También está referida a los métodos que mejoran la experiencia de uso durante el proceso de diseño o rediseño de un sitio". A esto se suma la noción de Donald Norman, para quien "la verdadera belleza en un producto va más allá de lo superficial, más allá de la fachada. Para que un producto sea verdaderamente bello, emocionante y agradable, debe cumplir una función, trabajar bien, ser usable y entendi ble" (2002, p. 36).

Si la aproximación de Nielsen al diseño de interfaces digitales, entre las cuales se encuentran los sitios web, proviene más del campo racional, el aporte de Norman tiene que ver con proporcionar una definición de usabilidad que estuviera estrechamente unida a la emoción de la experiencia estética.

Lo relevante de estos postulados es que en ambos se grafica la tendencia que últimamente se ha ido adoptando en el diseño y rediseño de sitios web, no sólo en términos de disposición espacial (en pantalla) de elementos textuales y gráficos, sino también respecto a la arquitectura de su información. Los usuarios ya no sólo leen en la red, sino que interactúan con la información que consumen y con las herramientas que la producen, impulsados en gran medida por el desarrollo de la web 2.0 a través de blogs, foros o plataformas sociales y sus aplicaciones. De esta manera, tanto las características visuales como la secuencia de interacciones hipertextuales, son partes integrales de la experiencia de $u_{s o^{2}}$ de un sitio.

En definitiva, al diseñar o rediseñar una página web no sólo debemos preocuparnos de que la interfaz sea usable y, por lo tanto, que esté estructurada bajo un orden lógico, sino que dicha interfaz contenga un diseño visual atractivo capaz de reflejar gráficamente la jerarquización del contenido. Esto último es de vital importancia pues, debido al desarrollo de las plataformas 2.0, actualmente la tendencia es que el contenido aumente vertiginosamente cada día. Así, tanto usabilidad como diseño visual redundarán en una experiencia de uso exitosa.
1 El Catálogo de objetos imposibles contenía más de 400 diseños de objetos de uso cotidiano reinterpretados en funcionalidades absurdas.

2 El término "experiencia de uso" fue popularizado luego de que Donald Norman se autodefinier como Arquitecto de Experiencia de Usuario en su paso por la empresa de computadores Apple en 1993. Originalmente usado para referirse a la interacción entre humanos y computadores, hoy el término se utiliza para hablar de las interacciones humanas, desde la relación con un aparato electrónico hasta la vivencia de un proceso de compra. Para efectos de este artículo, entenderemos el uso como la interacción que se produce entre quienes emiten y reciben las informaciones. Dicho rol, tal como se desarrollará aquí, se ha expandido notablemente con el advenimiento de las plataformas de producción de contenidos, tales como blogs o redes sociales. 


\section{LA EVIDENCIA DE LO RACIONAL}

Pensemos en un aeropuerto. El pasajero que viaja tiene poco tiempo para ejecutar sus movimientos. Debe realizarlos con certeza y a paso firme. Un sistema de información aeroportuaria mal diseñado puede fácilmente llevarlo a perder un vuelo o salir por el lugar equivocado. Si el lenguaje de signos falla, el viajero requerirá un esfuerzo mental y físico mucho mayor que el inicial, y podrá desorientarse fácilmente en su carrera por llegar a destino.

La usabilidad en sitios web funciona de manera similar a la señalética de un aeropuerto. Es decir, se trata de un sistema de signos orientativos que lleva a los usuarios a moverse entre página y página, a buscar y encontrar información, a orientarse en su propia interacción con el sitio a través de links, etiquetas o cualquier elemento que llame a una acción. En definitiva, hablamos de usabilidad cuando hablamos de la navegación de un sitio. Y hablamos de navegación cuando estamos en presencia de hipertextualidad, o sea, de la facultad de poder disponer de contenidos e informaciones que no se agotan en el mero texto escrito.

Según Nielsen (2003), la usabilidad es una condición necesaria para la supervivencia en la red. "Si un sitio es difícil de usar, los usuarios se van. Si la página de inicio no informa claramente sobre lo oferta de una compañía y lo que se puede hacer en dicho sitio, los usuarios se van. Si los usuarios se pierden en un sitio, se van. Si la información es difícil de leer o no responde a lo que los usuarios necesitan saber, éstos se van".

Steve Krug, en su libro No me hagas pensar, comparte la posición de Nielsen pero la lleva más allá: para él, el usuario que visita un sitio no debe ni quiere pensar. "Mientras sea humanamente posible, una página debe ser evidente al mirarla. Debe ser obvia. Explicarse por sí sola", (2000, p.11). Según Krug, el pensar nos requerirá más esfuerzo, y mientras más esfuerzo utilicemos para encontrar el contenido, estaremos menos dispuestos a leerlo e internalizarlo. De este modo, un sitio usable requerirá menos esfuerzo mental para recorrerlo tanto una primera vez como en ocasiones sucesivas, lo que implica que podrá ser navegado más rápidamente y dispondrá de una menor tasa de error y abandono.
Para Krug toda página debe contestar las cinco preguntas básicas que se haría un usuario al visitar un sitio web:

1) ¿Dónde estoy?

2) ¿Por dónde empiezo?

3) ¿Dónde está tal información?

4) ¿Qué es lo más importante de este sitio?

5) ¿Por qué lo llaman así?

Para explicar este concepto usaremos como ejemplo el cuento de Hansel y Gretel de los Hermanos Gri$\mathrm{mm}$. En esta clásica historia la idea era señalar el camino con migas de pan para ir siguiendo su rastro al volver a la casa de dulce cuando oscureciera. En el momento en que los pájaros se comen las migas de pan, ya no quedan rastros del camino. Los personajes se desorientan y no saben cómo volver. En el camino de Hansel y Gretel, lo que ocurrió fue que falló la navegación.

De acuerdo a James Kalbach en el libro Designing Web Navigation, (2007, sección 1.2), la navegación cumple diferentes funciones:

1) Permite acceder a la información: Al existir diferentes maneras, debemos ver el modelo más adecuado de acuerdo al contenido del sitio. Por ejemplo, es distinto acceder a la información por medio de una caja de búsqueda que desde la portada.

2) Informa del lugar de ubicación dentro del sitio ${ }^{3}$ : La navegación orienta a los usuarios, haciéndoles saber dónde están, a dónde van y qué hay en esa página.

3) Informa de qué se trata el sitio: La navegación debe ofrecer una visión semántica de qué se trata el sitio. Debe revelar lo que hay y lo que no hay, y si va por el camino correcto.

4) Identifica a la marca: Más allá de su logo, colores o tipografía, el sitio debe reflejar la marca del producto también en su navegación. La orientación de botones, etiquetas y textos debe reflejar la promesa de producto y servicio de la propia marca.

5) Refleja la credibilidad del sitio: Una buena navegación podrá disuadir y convencer a los usuarios de seguir un determinado camino. Mientras más creíble sea, más se podrá llegar a los usuarios.

6) Impacta en los costos: Una navegación correcta permitirá que los usuarios encuentren lo que buscan y eso tie 
ne una directa ventaja económica, pues un usuario que no encuentra lo que busca es siempre pérdida de dinero.

En síntesis, un sitio que carece de usabilidad no podrá ser navegado correctamente. Al no poder moverse fácilmente, no se puede encontrar lo que se busca. Esto se traduce en una mala experiencia de uso por parte del usuario, quien, probablemente, no querrá volver a visitar el sitio.

¿Cómo sabemos cuando un sitio es o no usable? Jakob Nielsen (2003) señala que las pruebas de usabilidad responden a cinco objetivos básicos:

- Aprendizaje: ¿Pueden los usuarios realizar tareas básicas en su primer encuentro con el sitio?

- Eficiencia: ¿Cuán rápido pueden los usuarios llevar a cabo esas tareas?

- Memoria: ¿Cuán fácil podemos reestablecer la productividad del sitio, una vez que los usuarios vuelven a visitarlo?

- Errores: ¿Cuántos errores cometen los usuarios? ¿Cuán severos son? ¿Cuán rápido se recuperan de esos errores?

- Satisfacción: ¿Cuán agradable es el diseño?

- Utilidad: ¿El diseño es funcional? ¿Cumple lo que los usuarios necesitan?

La medición de los parámetros de usabilidad del diseño o rediseño varía considerablemente. Desde sesiones de feedback informal de expertos a partir de zonificaciones y diagramas de contenido hasta pruebas de usabilidad más formales, tales como entrevistas con usuarios u observación participante.

De acuerdo al investigador Alan Cooper (2007, p.50) las técnicas de análisis cuantitativo usadas en la medición de estos parámetros responden a seis categorías:

1) Entrevistas con los responsables de la compañía, con el fin de poder entender los objetivos que se desean obtener tras el proceso de diseño o rediseño desde el punto de vista del negocio.

2) Entrevistas con expertos de la compañía, que manejen áreas sensibles en las que operará el producto que se desarrolle en el diseño o rediseño del sitio web.

3) Entrevistas con usuarios o clientes, entendiendo sus objetivos, frustraciones, procesos de toma de decisiones y dominio del ámbito y materia del producto.

4) Observación participante del uso que los usua- rios o clientes dan al sitio web.

5) Revisión de literatura relacionada con el ámbito del producto, tanto planes de marketing, estrategia, encuestas de usuarios, investigación de mercado, especificaciones técnicas, estadísticas y todo artículo que esté relacionado con el uso competitivo, tanto de los productos como del contenido que se desarrollará en el sitio web.

6) Estudio de prototipos o versiones existentes del actual producto o sitio web. Para este punto se recurre a evaluaciones heurísticas (informes de expertos) de las interfaces disponibles (en caso de un proceso de rediseño) o del prototipo visual del producto (si se trata de un primer diseño).

\section{LA EMOCIONALIDAD DE LO VISUAL}

La mentada web $2.0^{4}$ no sólo trajo consigo el surgimiento de las plataformas de producción de contenidos, tales como blogs o redes sociales ${ }^{5}$, sino que creó una nueva necesidad: el ordenamiento del volumen descontrolado de contenidos producido tanto por quienes ya estaban involucrados en la diseminación de información como por estos nuevos usuarios empoderados para poder administrar y emitir sus propios contenidos a través de estas nuevas plataformas. Así, el orden lógico emanado de las pautas de usabilidad descritas en una primera fase de la web, no se aplica. Había que lograr que la interfaz estructurada lógicamente llamara gráficamente la atención de los usuarios. En otras palabras, que la interfaz no sólo fuera racionalidad, sino que también fuera capaz de emocionar (y agradar) a través del diseño. Esto porque, a través de esta emoción, los usuarios se lograrían sentir atraídos y podrían interpretar correctamente el orden racional de este nuevo volumen de contenidos.

Pensemos en los monitores del computador. En un principio eran en blanco y negro. Dichos monitores servían perfectamente para las funciones que habían sido creados: eran usables. Sin embargo, los fabricantes insistieron con añadirle color a las pantallas. Podrían no haber tenido ninguna razón aparente, ya que tanto los en blanco y negro como los de color cumplirían exactamente las mismas funciones. Sin embargo, sí había un fundamento: el color apelaba a la reacción emocional de los clientes, pues era más atractivo un monitor en colores que uno en blanco y negro.
4 De acuerdo al investigador Tim 0'Reilly (2007) la web 2.0 se ha definido como una revolución en la industria tecnológica causada por el auge que ha tenido Internet como una plataforma y el intento por entender el éxito de dicha plataforma. Según O'Reilly, la regla principal dentro de esta fórmula del éxito de la web es la construcción de aplicaciones que permiten que la gente haga mejor uso del Internet.

5 Instrumentos tales como Blogger, Facebook, You Tube - Flickr se califican como parte de las plataformas de producción de contenidos de la web 2.0. 
6 Los estudios de la emoción de Norman, conducidos en el departamento de Psicología de la Universidad de Northwesten en Estados Unidos se encuentran descritos en el capítulo 3 del libro Emotional design: why we love lor hate everyday things. Allí el autor desarrollo como cada nivel de aproximación al objeto requiere un estilo de diseño diferente.
Con este ejemplo, Donald Norman, en su libro Diseño emocional ha introducido uno de los análisis más interesantes en la conexión entre visualidad y emoción, y que es extensible a las plataformas digitales. Según el autor, los seres humanos imprimimos significados a los objetos a los que nos enfrentamos en nuestra vida cotidiana. Tales significados muchas veces están asociados a un valor, incluso a un precio. Sin embargo, estos objetos cargados de significación no los usamos para jactarnos con ellos, sino porque nos evocan sentimientos positivos, recuerdos agradables o, muchas veces, porque son un reflejo de nosotros mismos, una señal de identidad.

Si pensamos en la cognición como fría, humana y lógica, la emoción sería el componente cálido, animal e irracional. Lo que históricamente ha sido una primacía del pensamiento lógico sobre el emocional, hoy es una mezcla inseparable. "Las emociones son una parte necesaria de la cognición. Todo lo que hacemos, todo lo que pensamos está teñido de emoción, mucha de la cual es subconsciente", señala Norman (2004, p. 7).

El investigador ha conducido estudios que lo han llevado a establecer que hay tres dimensiones en que el ser humano se aproxima a un objeto a nivel emocional: la visceral, la del comportamiento y la reflexiva ${ }^{6}$ (2004, p.31). A partir de esta aproximación al objeto, el autor ha sido capaz de aseverar que un diseño atractivo hace que la gente se sienta bien, y una persona que se siente bien piensa más creativamente. En consecuencia, un diseño seductor le permitirá a los usuarios encontrar las soluciones a sus desafíos mucho más fácilmente y, por lo tanto, incrementará la usabilidad de un producto o, en este caso, de un sitio web.

Volvamos al ejemplo del aeropuerto. Para un pasajero que va apurado un pictograma que retrate la salida, otro el sector de las maletas y quizás uno que refleje el símbolo de aduana, permitirá que aunque el viajero no entienda el idioma en que están los textos que acompañan a dichos pictogramas, comprenda cómo llegar a destino. La visualidad de éstos ayuda a disminuir la probabilidad de que el pasajero se pierda a la hora de tomar un vuelo, pues apela a la memoria colectiva del usuario, quien ya sabe qué significa cada uno de esos símbolos.

En el complejo sistema de navegación de la web, los componentes gráficos permitirán añadirle el componente emocional de la experiencia a la racionalidad del uso. La visualidad permite así que el sitio "hable" no sólo en lenguaje de texto, sino también en el visual. Y, por eso, tal como se ordenan los contenidos textuales, se deberá estructurar correctamente el contenido visual.

\section{LO PRIMERO ES LO MÁS IMPORTANTE}

Jennifer Tidwel en Designing Interfaces argumenta que el concepto de jerarquía visual juega un rol en todas las formas de diseño gráfico. "Poniéndolo simplemente, el contenido más importante debe ser el más destacado, mientras que el menos importante, debe destacarse menos. Los títulos deben verse como títulos y el contenido secundario debe verse como tal, en definitiva, un lector debe ser capaz de deducir la estructura de información de una página desde su diseño", (Tidwell, 2006, p 58). Es por eso que las decisiones de diseño que finalmente repercutirán en la jerarquía visual del sitio, no deben ser hechas al azar.

La primera tarea del diseño web, en consecuencia, será crear una jerarquía visual fuerte y consistente, donde los elementos importantes sean enfatizados y el contenido esté ordenado lógica y asertivamente, tanto en sus códigos textuales como no textuales. Siguiendo la definición de estos códigos que nos da Javier Royo en el libro Diseño digital (2004, p. 173-174), los códigos lingüísticos gráficos con los que generamos (escribimos, programamos, diseñamos), leemos (escuchamos, vemos) y participamos (interactuamos) en el ciberespacio se pueden dividir en dos:

1) Los códigos visuales: la escritura alfabética (alfabeto: tipografía), la escritura no alfabética (íconos, esquemas) y la imagen fija (ilustración, fotografía);

2) Los códigos secuenciales: la imagen en movimiento y la hipertextualidad.

Ahora bien, si el sistema visual me lo explica claramente, es mucho más fácil saber dónde nos encontramos en el sitio, a qué página vamos, a dónde me lleva este link, cuánta información existe sobre este tema o cómo termino esa transacción. Y para esto, tal como se- 
ñala Krug (2000) "una de las mejores maneras de lograr captar a los esquivos usuarios es asegurar que los elementos de una página retraten clara y exactamente las relaciones entre ellos" (p.31).

De esta manera, la organización visual se logra reconociendo similitudes y diferencias entre los objetos gráficos. Por ejemplo, si en un mapa de Chile todas las ciudades estuvieran descritas en un mismo tamaño de texto, sería muy difícil saber cuáles son las capitales regionales o las con mayor población. Si le asignamos a Santiago la tipografía de mayor tamaño, inmediatamente podremos deducir que se trata de la capital o al menos una ciudad con mayor relevancia a las demás. Como las relaciones visuales entre los objetos nos permitirán interpretar lo que vemos, establecer estas relaciones nos permitirá, además, ir contando una historia acerca de lo que estamos viendo visualmente.

Ahora bien, a la hora de jerarquizar, existen cinco pautas que regulan la construcción de una historia visual en la mente del usuario:

1. la proximidad - un elemento que se aleja del grupo, es percibido como un elemento nuevo.

2. la similitud - elementos de un mismo tamaño, forma, color, posición o textura son percibidos como partes de un mismo grupo.

3. la continuidad - de elementos similares, aunque distintos en forma y tamaño.

4. la cercanía - la percepción tiende a agrupar elementos que se encuentran muy cercanos entre sí.

4. y la asimilación - tendemos a asimilar más fácilmente aquellos objetos que ya conocemos.

Cuando miramos un diseño, en particular el web, tendemos a notar las relaciones entre los objetos expuestos. Dichas relaciones nacen de las diferencias, por ejemplo, si un titular es rojo y un texto es azul, sabremos que se trata de dos objetos diferentes. Así, podremos agruparlos en función del color, la textura, la forma, la posición o el tamaño.

De acuerdo a estos postulados, resulta aún más relevante saber que la posición, color, estilo o tamaño son elementos que, junto al contenido textual, definen la eficiencia comunicativa del sitio. El lenguaje visual es tan importante como el lenguaje de texto y, como tal, de- bemos prestarle atención con una clara jerarquización de los elementos que lo componen. Ésta debe "crear un foco de interés que atraiga la atención del usuario, dar un sentido de orden y equilibrio, y establecer un patrón de movimiento que guíe al usuario a través de la composición" (Wroblewski, 2002, p.77). En definitiva, necesitamos contar una historia a través de los elementos gráficos.

Bruce Tognazzini en su artículo Principios de diseño de interacción (2003) ha resumido las normas que regulan el diseño e implementación de interfaces gráficas efectivas, aplicándolo al campo del diseño web. En términos generales, señala que:

Las interfaces efectivas son visualmente comprensibles y permiten errores por parte del usuario, dándole una sensación de control. Los usuarios ven rápidamente el alcance de las opciones y comprenden como alcanzar sus metas y realizar su trabajo. Las interfaces efectivas ocultan al usuario el funcionamiento interno del sistema. El trabajo se guarda continuamente y con la opción de deshacer en todo momento cualquier paso que se haya dado. Las aplicaciones y servicios efectivos realizan el máximo trabajo requiriendo la mínima información del usuario.

\section{UNIÓN ENTRE RAZÓN Y EMOCIÓN}

Pensar en una experiencia de uso exitosa hoy es hablar de una combinación entre la racionalización de la usabilidad y la emocionalidad de lo visual. El usuario se ha ido empoderado para interactuar y crear sus propios contenidos, generando un incremento considerable en el volumen de éstos. Por esta razón, debemos procurar que la experiencia de visitar un sitio sea una unión entre la razón y la emoción, entre la lógica y la visualidad.

El autor Jesse James Garrett en el libro The Elements of User Experience propone un modelo donde los elementos que componen la experiencia de uso de un sitio se encuentran desagregados en etapas. Cumplir cada una de estas etapas, según él, asegurará una correcta experiencia de uso.

El modelo de Garrett desagrega dicha experiencia en 5 planos: 
Modelo de experiencia de usuario de J.J. Garrett

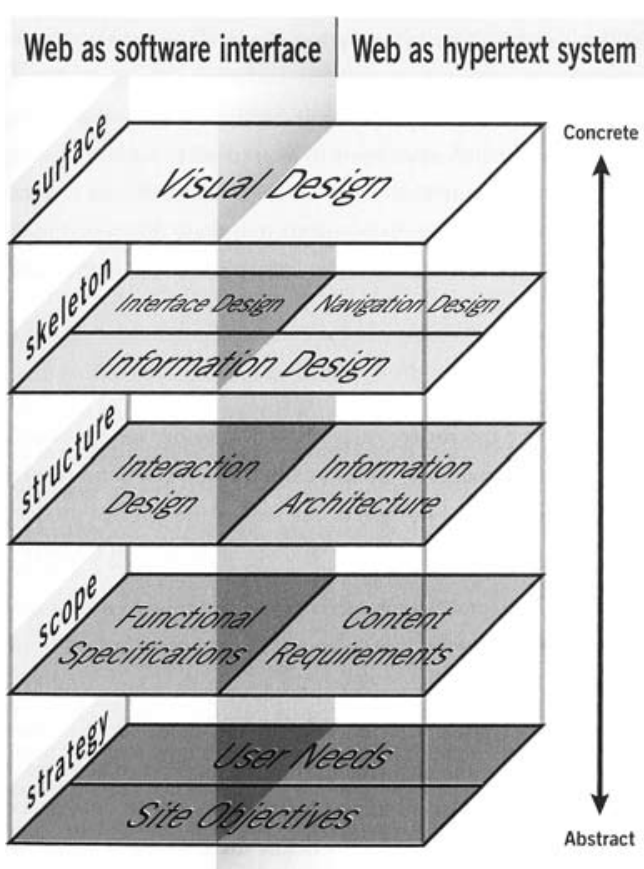

fuente: Garrett, J.J. (2003).

1) El Plano de la Estrategia: Aquí se determina y define al sitio en función de los objetivos de los creadores y de lo que los usuarios quieren obtener de la página.

2) El Ámbito del Proyecto: Aquí se define las características y las funcionalidades del sitio.

3) El Plano Estructural: En este se define de dónde vienen los usuarios y a dónde van, es decir, se preocupa de profundizar en las características y funcionalidades en las que se inserta el sitio, vistas en el punto anterior.
4) El Plano del Esqueleto: Esto se refiere a la ubicación de los botones, las etiquetas, las fotografías y los bloques de texto. En el esqueleto se ubican estos elementos para sacar el máximo provecho y efectividad de ellos.

5) El Plano Superficial: Es la capa más externa y se refiere al contenido textual y gráfico del sitio. Desde las imágenes hasta los textos, el plano superficial habla del diseño visual en sí mismo.

A partir de estos cinco planos, que van de lo más abstracto a lo más concreto, Garrett señala que se puede definir un marco conceptual que permita delinear problemas y soluciones acerca de la experiencia de uso. Cada uno de estos planos descritos depende del siguiente: la superficie dependerá del esqueleto, éste de la estructura, la estructura del ámbito del proyecto, y éste no se podrá definir sin una estrategia. Por consiguiente, las decisiones deben hacerse en el orden ya descrito, puesto que, por ejemplo, un cambio en la estrategia afectará todo el proyecto.

\section{CONCLUSIONES}

En conclusión, es verdad que el orden y la estructura de un proyecto será lo que guiará su proceso. Pero un diseño o rediseño que no considere desde el comienzo una coexistencia de factores racionales (usabilidad) con los emocionales (visualidad) no podrá asegurar una experiencia de uso exitosa. El objetivo es simple. Tal como señala Thomas Baekdal en La batalla entre usabilidad y experiencia de uso (2006), visitar un sitio es "crear felicidad". En otras palabras, la idea es que los usuarios se sientan felices, antes, durante y después de haber usado el producto. Sólo así tendremos experiencias de uso exitosas y un usuario contento que seguramente volverá una y otra vez, asegurando la sobrevivencia, o mejor aún, el crecimiento, del sitio en la web. 


\section{REFERENCIAS}

Baekdal, T. (2006). The Battle Between Usability and User Experience. Recuperado el 19 de junio de 2006 desde http://www.baekdal.com/

Beaird, J. (2007). The Principles of Beautiful Web Design. Australia: SitePoint.

Cooper, A.; Reimann, R. y Cronin, D. (2007). About Face 3: The Essentials of interaction Design. Estados Unidos: Wiley Publisihing.

Garrett, J. J. (2003). The Elements of User Experience. Estados Unidos: New Riders Publishing.

Kalbach, J. (2007). Designing Web Navigation. Estados Unidos: O’Reilly Media

Krug, S. (2000). Don't Make me Think. Estados Unidos: New Riders Publishing.

Lynch, P. y Horton, S. (2002). Web Style Guide: Basic Design Principles for Creating Web Sites. Estados Unidos: Yale University Press.

Mijksenaar, P. (2001). Diseño de la Información. México: Ediciones Gustavo Gili.

Nielsen, J. (2000). Designing Web Usability. Estados Unidos: New Riders Publishing

Nielsen, J. (2003, 25 de agosto). Usability 101: Introduction to Usability. Recuperado el 25 de agosto de 2003 desde http://www.useit.com/alertbox/20030825.html
Norman, D.A. (1990). Design of Everyday Things. Estados Unidos: First Doubleday.

Norman, D. A. (2002). Emotion and design: Atractive things work better. Interactions Magazine, ix (4), 36 42.

Norman, D. A. (2004). Emotional Design: Why We Love (or Hate) Everyday Things. Estados Unidos: Basic Books.

O'Reilly, T. (2007). Web 2.0 Compact Definition: Trying Again. Recuperado el 6 de enero de 2008 desde http://radar.oreilly.com/2006/12/web-20-compactdefinition-tryi.html

Royo, J. (2004). Diseño Digital. España: Ediciones Paidós Ibérica.

Tidwell, J. (2006). Designing Interfaces. Estados Unidos: O’Reilly Media.

Tognazzini, Bruce (2003). First Principles of Interaction Design. Recuperado 2 de junio de 2008 desde http:// www.asktog.com/basics/firstPrinciples.html

Wrobleski, L. (2002). Site-seeing: A visual Approach to Web Usability. Estados Unidos: Hungry Minds. 